\title{
Geometry-Dependent Electronic Properties of Highly Fluorescent Conjugated Molecules
}

\author{
Shu-Chun Yang, ${ }^{1}$ W. Graupner, ${ }^{2}$ S. Guha, ${ }^{3}$ P. Puschnig, ${ }^{4}$ C. Martin, ${ }^{1}$ H. R. Chandrasekhar, ${ }^{1}$ M. Chandrasekhar, ${ }^{1}$ \\ G. Leising, ${ }^{5}$ C. Ambrosch-Draxl, ${ }^{4}$ and U. Scherf ${ }^{6}$ \\ ${ }^{1}$ Department of Physics and Astronomy, University of Missouri, Columbia, Missouri 65211 \\ ${ }^{2}$ Department of Physics, Virginia Tech, Blacksburg, Virginia 24061-0435 \\ ${ }^{3}$ Department of Physics, Marquette University, Milwaukee, Wisconsin 53201-1881 \\ ${ }^{4}$ Institut für Theoretische Physik, Universität Graz, Graz, A 8010 Austria \\ ${ }^{5}$ Institut für Festkörperphysik, Technische Universität Graz, Graz, A 8010 Austria \\ ${ }^{6}$ Max-Planck-Institut für Polymerforschung, Mainz, D 55128, Germany
}

(Received 19 July 1999)

\begin{abstract}
We present a combined experimental/theoretical study of the electronic properties of conjugated paraphenylene type molecules under high pressure up to $80 \mathrm{kbar}$. Pressure is used as a tool to vary the molecular geometry and intermolecular interaction. The influence of the latter two on singlet and triplet excitons as well as polarons is monitored via optical spectroscopy. We have performed band structure calculations for the planar poly (para-phenylene) and calculated the dielectric function. By varying the intermolecular distances and the length of the polymer repeat unit the observed pressure effects can be explained.
\end{abstract}

PACS numbers: 78.66.Qn, 31.50.+w, 71.23. $-\mathrm{k}, 71.38 .+\mathrm{i}$

Conjugated polymers are ideal candidates for electroluminescent devices [1] which require certain electronic properties in terms of charge and energy transport as well as photon emission. Isolated conjugated molecules are low dimensional electronic systems. However, in the solid state, the electronic properties depend on threedimensional interactions [2,3]. These interactions lead to completely new transitions due to collective states such as aggregates [2] or to shifts of the energy levels of single molecules due to changes in the molecular geometry [4].

The focus of this study is the influence of molecular geometry and intermolecular interaction on the electronic properties of films of methylated laddertype poly(paraphenylene) ( $m$-LPPP) [5] — a material of very low defect content [6], used for blue and white light emitting devices [7]. The small inhomogeneous broadening in $m$-LPPP makes the electronic spectra easily detectable fingerprints for the involved states.

A diamond anvil cell was used for hydrostatic pressure studies [8,9]. The photomodulation spectra were taken in an optical access cryostat at $80 \mathrm{~K}$. The $457.9 \mathrm{~nm}$ line of an Ar-ion laser was the pump beam, modulated by a mechanical chopper. A halogen lamp was used for the probe beam, which passed through the sample and was then dispersed by a SPEX 1702 single stage monochromator to be detected by photovoltaic detectors with builtin preamplifiers. The small changes $\Delta T$ in transmission were picked up by a lock-in amplifier referenced to the chopper. All photomodulation spectra are $\Delta T$ divided by the transmission $T$. In Fig. 1 we show the electronic states and transitions in conjugated molecules. Absorption of light occurs via creation of a singlet excited state $S_{1}$, as well as phonons. After photoexcitation, deexcitation occurs via several mechanisms: Nonradiative recombination (NR) leading to a depopulation of $S_{1}$ via creation of phonons; radiative recombination of $S_{1}$ leading to photoluminescence (PL); population of the lowest lying triplet state $T_{1}$ via intersystem crossing (ISC) from $S_{1}$, which can be probed by $T_{1} \rightarrow T_{\mathrm{N}}$ triplet-triplet absorption (TT); dissociation of the $S_{1}$ (= bound electron-hole pair) into a free electron hole pair, which creates one positive and one negative polaron, giving rise to intrapolaron absorption $(P)$. Population of triplet and polaron states leads to a small depopulation of the ground state, causing photobleaching (PB) of the absorption. We omit the vibronic structure of the TT and the polaron transitions for clarity. Involvement of no phonon is expressed at 0-0-transition, one phonon as 0 -1-transition, and so forth.

The $\Delta T / T$ spectra monitor TT and $P$ absorption as well as PB. In pure or as-prepared $m$-LPPP films no signatures of polarons are found in the $\Delta T / T$ spectra [10]. This is due to the low defect content, as in "improved PPV" [11] - polaron formation and stabilization are favored by the presence of defects [10]. We introduced defects via photo-oxidizing the $m$-LPPP with $50 \mathrm{~mW}$ of the $351.1 \mathrm{~nm}$ Ar-ion laser line for about $8 \mathrm{~min}$ in air. In Fig. 2 we show

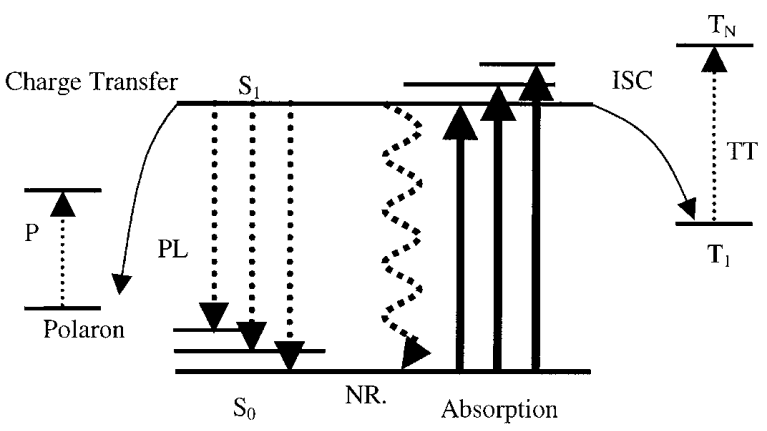

FIG. 1. Scheme of electronic states and transitions in conjugated molecules such as $m$-LPPP (see text). 


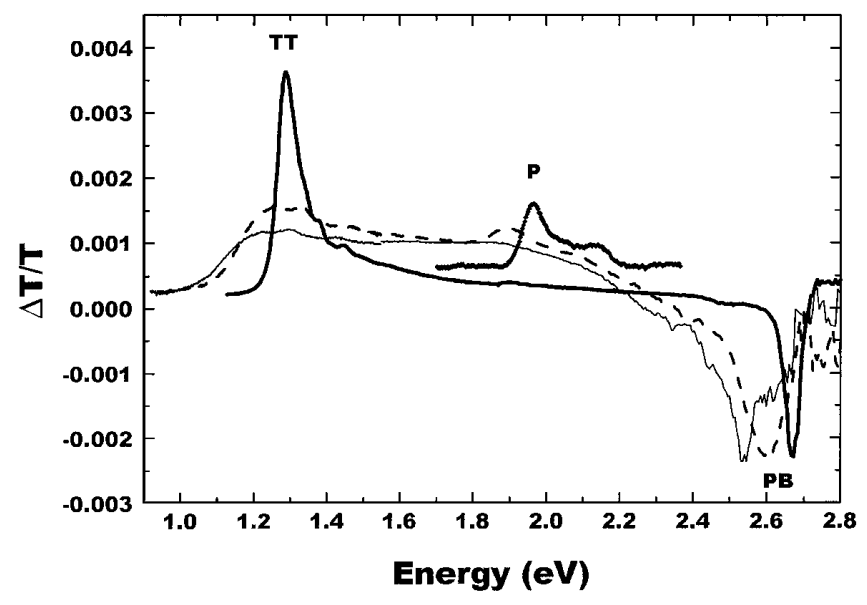

FIG. 2. Photomodulation spectra of photo-oxidized $m$-LPPP for 38 (dashed line) and 66 (thin solid line) kbar. Chopper frequency $=145 \mathrm{~Hz}$, sample temperature $=80 \mathrm{~K}$. Thick solid lines: 1 bar data of a pure $m$-LPPP film and the polaron absorption $(P)$ of a photo-oxidized film.

the $\Delta T / T$ spectra of a photo-oxidized $m$-LPPP film for various pressures. The polarons, as well as the TT and PB transitions, are all observed at low pressure; the polaron absorption is difficult to detect at $66 \mathrm{kbar}$. In order to ensure that the introduction of defects does not dramatically alter the electronic properties we did control experiments with as-prepared films. Aside from the polaron transition the spectra of pure and photo-oxidized films did not vary strongly. The pressure dependencies of the PB and TT features are identical for both types of films.

The application of hydrostatic pressure increases intermolecular interaction and changes in the molecular geometry without any chemical changes in the materials. Hence, pressure is a "clean" way to investigate the influence of molecular geometry. We deduce the nature of the geometry changes from their influence on the electronic transitions by comparing the experimental results to calculations. Varying pressure also allows us to see which transitions/ states are connected to each other, e.g., via a population/ depopulation mechanism and, which states are not coupled, i.e., show different changes of the spectra under pressure.

The influence of pressure on optical spectra of $m$-LPPP is summarized in Table I. We observe a redshift and a

TABLE I. Pressure dependence of absorption (abs.), PL emission, and photoinduced absorption spectra.

\begin{tabular}{lcc}
\hline \hline \multicolumn{1}{c}{ Transition } & $E(0)[\mathrm{eV}]$ & $\alpha[\mathrm{meV} / \mathrm{kbar}]$ \\
\hline 0-0 PL & 2.67 & -2.5 \\
0-1 PL & 2.50 & -2.1 \\
0-0 abs. & 2.73 & $-3.8^{\mathrm{a}}$ \\
PB film & 2.68 & -2.1 \\
Polaron $(P)$ & 1.97 & -2.1 \\
TT $\left(T_{1} \rightarrow T_{N}\right)$ & 1.28 & -1.2 \\
\hline \hline
\end{tabular}

${ }^{a}$ The pressure dependence of the 0-0 absorption peak of $m$-LPPP has also a nonlinear component. broadening of all spectra with increasing pressure. The shift of the transition energy at pressure $p$ is described as $E(p)=E(0)+\alpha p$ with $E(0)$ being the transition energy at $p=0$ and $\alpha$ being the pressure coefficient. The $\alpha$ for PB and 0-1 PL are practically identical. These two transitions are not influenced by any competing ones- the 0-0 PL, e.g., is influenced by the absorption spectrum since the Stokes shift in $m$-LPPP is $<0.1 \mathrm{eV}$. The effective conjugation depends on the degree of overlap of the $\pi$-electron wave functions [12]. Therefore, the observed shifts under pressure can be explained by a higher degree of conjugation due to the stronger overlap of $\pi$ electrons, i.e., a higher degree of planarity [4,12] or shorter inter-ring bonds [13].

One way of increasing the degree of conjugation in short conjugated molecules is simply to increase their length, i.e., adding more conjugated bonds [14]. Upon increasing the conjugation length the energy shift of the TT transition was calculated to be smaller than the shift of the singlet-singlet transition because the triplet states are localized at one phenyl ring [15]. This localization is not due to extrinsic disorder but an intrinsic property of the triplet wave function induced by the exchange term [16]. Therefore, our result of the small TT-absorption shift of $-1.2 \mathrm{meV} / \mathrm{kbar}$ is consistent with the theoretical prediction: As pressure varies the degree of conjugation the triplet transitions are less affected than the singlet transitions (absorption, PL, PB). Polarons, however, are three to four times more extended [17] than the triplet. Therefore their pressure behavior resembles that of the singlet states - their respective $\alpha$ is close to the singlet values, which is in excellent agreement with the behavior observed for oligophenyls [18].

The $0-0$ absorption shift is very large $(-3.8 \mathrm{meV} / \mathrm{kbar})$, and contains a nonlinear component in pressure [8]. The latter may arise because absorption probes all the chain segments in the film. If the relative shift in transition energies under the influence of pressure is greater for smaller segments, this will lead to the observed stronger shift. The PL, TT, PB, and P signals all originate from the chains with the longest effective conjugation, i.e., only the sites lowest in transition energy, to which excited states migrate. Absorption reflects all the different sites and is therefore much broader [8]. It shows how all the sites evolve under pressure.

In order to model the intermolecular interaction we performed three-dimensional (3D) band structure calculations based on density functional theory (DFT) $[19,20]$ for planar PPP (Fig. 3). By varying the intermolecular distances $(a, b)$ and the length of the interring bond $(c)$, pressure effects were simulated. The second derivative of the total energy with respect to the lattice constants is related to the compressibility of the system. Our computations assume three-dimensional periodicity of the structure-hence the solutions of the Kohn-Sham equations are Bloch waves. In that respect our method differs significantly from the quantum chemical approach used in [2] since we investigate the 


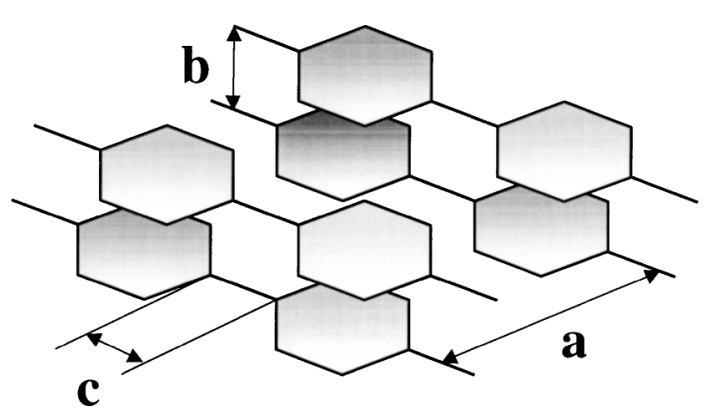

FIG. 3. Three-dimensional arrangement of planar poly(paraphenylene) molecules; $a$ and $b$ denote the separation of the molecular axes, $c$ the length of the single bond between the phenyl rings.

influence of intermolecular interactions in an extended $3 D$ system. Quantities involving excited states such as the dielectric tensor are handled $a b$ initio by our approach. All calculations were carried out with the full-potential linearized augmented plane wave (FP-LAPW) method utilizing the WIEN97 code [21]. For exchange and correlation effects the generalized gradient approximation according to Perdew et al. has been applied [22]. Optical absorption is determined by the imaginary part of the dielectric function $\operatorname{Im} \varepsilon$, obtained by ab initio computations based on the knowledge of the single-particle orbitals [23] and energies approximated by the solutions of the Kohn-Sham equation.

Figure 4(a) shows a linear redshift of Im $\varepsilon$ for decreasing $c$ but no broadening. The shift is a result of the increasing bandwidth of the delocalized valence and conduction bands along the polymer axis when the inter-ring bond is shortened. This is equivalent to a stronger overlap of the $\pi$ electrons, leading to a lowering of the electronic transition energies, an effect expressed as increased degree of conjugation. The width of $\operatorname{Im} \varepsilon$, however, remains almost constant, because the main contributions to the dielectric function arise from the flat regions in reciprocal space perpendicular to the polymer axis (high joint density of states), which remain unaltered. When changing $c$, we let the bond lengths and angles relax via $a b$ initio force calculations for each value of $c$. From the total energy as a function of the lattice constant we obtained the bulk modulus for an infinitely large PPP crystal to be $3 \times 10^{11} \mathrm{~N} / \mathrm{m}^{2}$ in good agreement with experimental values [24] for terphenyl.

A reduction in $a$ as well as in $b$ results in a slight blueshift of Ime and in a strong broadening. The blue shift as well as the broadening are due to intermolecular interactions that result in an increased bandwidth of the localized valence and conduction bands perpendicular to the polymer. The stronger overlap of the wave functions on two neighboring chains due to decreasing interchain distance results in a higher dispersion of the bands in that direction and the joint density of states for the optical transition is broadened - an effect clearly detectable in all transitions.

The redshift and the broadening of the absorption spectra of $m$-LPPP under high pressure can be understood when
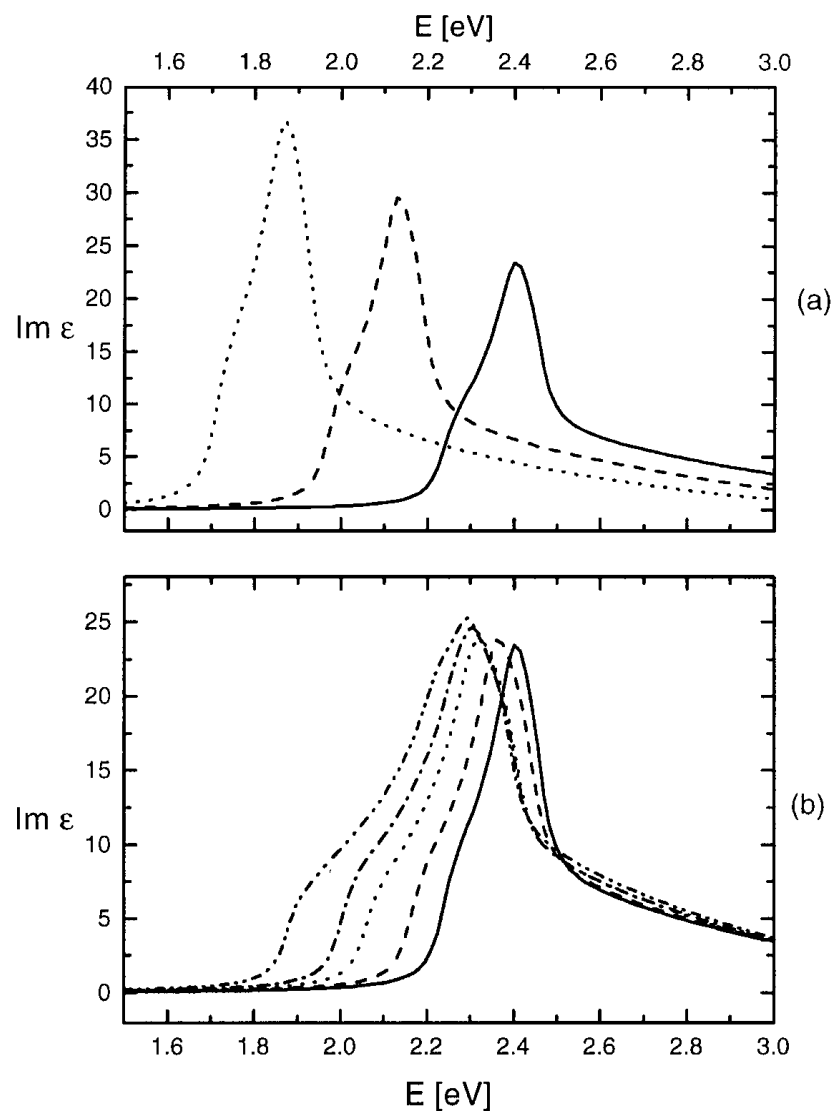

FIG. 4. (a) Imaginary part of the dielectric function ( $\operatorname{Im} \varepsilon$ ) for different values of $c: c=1.38,1.43,1.49 \AA$ (from left to right): ( $a=4.5 \AA, b=5.0 \AA$ ); (b) Im $\varepsilon$ for different $c$ and $b$, from left to right: $(c, b)=(1.45 \AA, 4.0 \AA),(1.46 \AA, 4.3 \AA),(1.47 \AA$, $4.5 \AA),(1.48 \AA, 4.8 \AA),(1.49 \AA, 5.0 \AA)(a=4.5 \AA)$. $(c, b)$ are chosen in such a way as to describe quantitatively the experimentally observed PB spectra shown in Fig. 2. We superimposed a Gaussian broadening of $0.1 \mathrm{eV}$ on all spectra. For $a, b, c$ see Fig. 3.

combining both mechanisms described above: We have chosen $(c, b)$ in Fig. 4(b) in such a way as to describe quantitatively the experimentally observed PB spectra shown in Fig. 2 (i.e., broadening and redshift). From the calculated bulk modulus we computed a redshift of $-2.1 \mathrm{meV}$ per kbar for the ground state absorption in $m$-LPPP, which is in excellent agreement with the experimental values (Table I).

To find out how intermolecular interaction stabilizes or destabilizes localized states we measured $\Delta T / T$ of the photo-oxidized $m$-LPPP film at the spectral positions of PB and TT vs chopping frequency $f$ at 38 and $65 \mathrm{kbar}$ (Fig. 5). If the $(\Delta T / T)$ signal is due to states with one particular time constant $\tau$, the relation $(\Delta T / T)=[1+$ $\left.(2 \pi f \tau)^{2}\right]^{-1 / 2}$ holds [25]. $(\Delta T / T)$ shows no dependence on $f$ if $\tau$ is much smaller than $1 / f$. In general a more complex relation is found since the real system often shows a $\tau$ distribution [25]. In all cases the following rules hold: (1) If two signals originate from states with the same $\tau$ distributions, the same frequency dependence is observed 


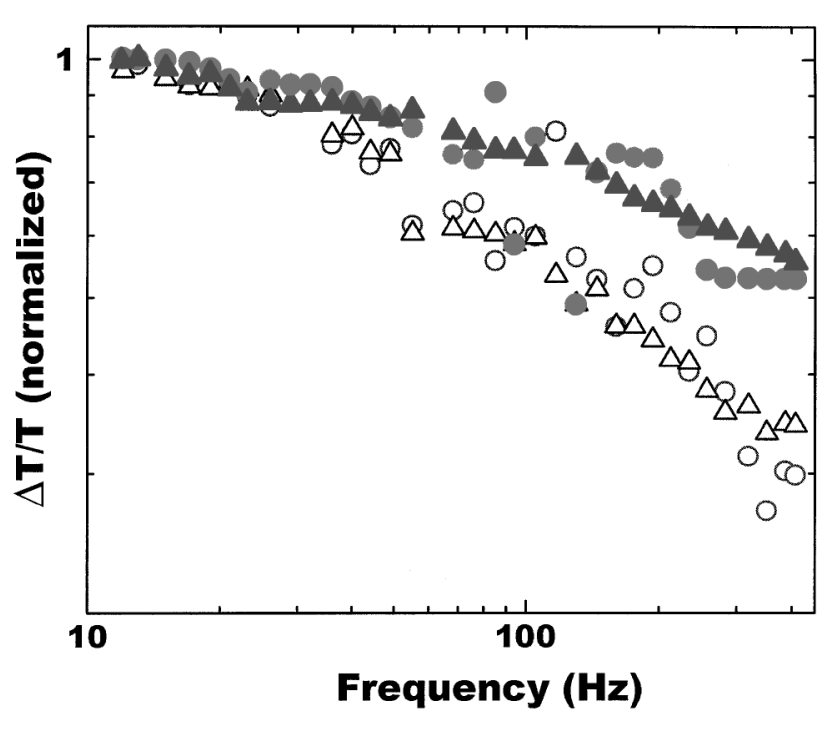

FIG. 5. Photobleaching (empty circles) and triplet triplet (empty triangles) signal at $38 \mathrm{kbar}$ and photobleaching (full circles) and triplet triplet (full triangles) signal at $65 \mathrm{kbar}$ versus chopper frequency. The data have been renormalized to yield a value of unity when extrapolated to $f=0 \mathrm{~Hz}$.

for these signals, (2) a weaker dependence of the signal in a given $f$ range indicates shorter $\tau$. In that sense we draw three conclusions. (A) Since the TT and PB signal show identical frequency behavior they have identical $\tau$ distributions. Thus, the electrons and holes populating the triplet states are the same, which are missing in the ground state giving rise to $\mathrm{PB}$. (B) When going from 38 to $65 \mathrm{kbar}$ the frequency dependence becomes more flat: The $\tau$ of the involved states decreases. Smaller $\tau$ correspond to less stable states - the increase in intermolecular interaction destabilizes triplets and polarons, as predicted in [26]. The decrease in $\tau$ also accounts for a smaller TT and PB signal at higher pressures (see Fig. 3). (C) The fact that the frequency dependence of both PB and TT signal changes in the same way upon a pressure change supports conclusion (A).

In conclusion, we have shown a redshift and broadening for the electronic singlet, triplet-exciton, and polaron transitions under pressure. The pressure coefficient for ground state absorption of $-2 \mathrm{meV} / \mathrm{kbar}$ is in excellent agreement with our calculations. The smaller value for triplets is due to their small spatial extent compared to singlets. The DFT calculations reveal that a decrease of intramolecular distances leads to a redshift, and a decrease of intermolecular distances leads to a broadening of the spectra. The observed $\mathrm{PB}$ is due to a population of the $T_{1}$ triplet state.
The increase in intermolecular interaction destabilizes localized states, as predicted in [26].

Supported by the University of Missouri Research Board, OeNB Project No. 6608, the vector-computer facilities at the University of Graz. We acknowledge the interaction with I. D'Amico, C. A. Ullrich, B. Ullrich, and V. Ashkenazy.

[1] J. H. Burroughes et al., Nature (London) 347, 539 (1990).

[2] J. Cornil et al., J. Am. Chem. Soc. 120, 1289 (1998).

[3] P. Puschnig and C. Ambrosch-Draxl, Phys. Rev. B 60, 7891 (1999).

[4] S. Guha et al., Phys. Rev. Lett. 82, 3625 (1999).

[5] U. Scherf and K. Müllen, Makromol. Chem. Rapid Commun. 12, 489 (1991).

[6] W. Graupner et al., Phys. Rev. B 54, 7610 (1996).

[7] S. Tasch et al., Appl. Phys. Lett. 71, 2883 (1997).

[8] S. Guha et al., Phys. Status Solidi 11, 77 (1999).

[9] S. Webster and D. N. Batchelder, Polymer 37, 4961 (1996).

[10] W. Graupner et al., Synth. Met. 69, 419 (1995).

[11] K. Pichler et al., Synth. Met. 55, 230 (1993).

[12] J. Roncali, Chem. Rev. 97, 173 (1997).

[13] The length of the inter-ring bond in biphenyl changes from 1.482 to $1.473 \AA$ Apon increasing pressure from 0 to $2 \mathrm{kbar}$ [P. Puschnig and C. Ambrosch-Draxl (unpublished)].

[14] J. L. Bredas et al., J. Am. Chem. Soc. 105, 6555 (1983).

[15] D. Beljonne et al., J. Am. Chem. Soc. 118, 6453 (1996); J. Cornil et al., J. Chem. Phys. 103, 842 (1995); E. J. W. List et al., Phys. Rev. B 61, 10807 (2000).

[16] D. A. dos Santos et al., Chem. Phys. 227, 1 (1998).

[17] E. Zojer et al., Phys. Rev. B 59, 7957 (1999).

[18] R. K. Khanna et al., Chem. Mater. 5, 1792 (1993).

[19] P. Hohenberg and W. Kohn, Phys. Rev. 136, 864 (1964); W. Kohn and L. J. Sham, Phys. Rev. 140, A1133 (1965).

[20] R. O. Jones and O. Gunnarsson, Rev. Mod. Phys. 61, 689 (1989).

[21] P. Blaha et al. WIEN97, A Full Potential Linearized Augmented Plane Wave Package for Calculating Crystal Properties, (Karlheinz Schwarz, TU Wien, Austria, 1999), ISBN 3-9501031-0-4.

[22] J. P. Perdew et al., Phys. Rev. Lett. 77, 3865 (1996).

[23] C. Ambrosch-Draxl et al., Phys. Rev. B 51, 9668 (1995).

[24] H. Landolt and R. Börnstein, Zahlenwerte und Funktionen aus Physik, Chemie, Astronomie, Geophysik und Technik (Springer, Berlin, 1957), 6th ed., Vol. 2.

[25] W. Graupner et al., Phys. Rev. Lett. 77, 2033 (1996).

[26] P. Vogl and D. K. Campbell, Phys. Rev. Lett. 62, 2012 (1989). 